\title{
Placental thickness in 2D prenatal ultrasonographic examination
}

Iwona Strzelecka¹, Filip F. Karuga², Bartosz Szmyd³ ${ }^{3}$ Aleksandra Walter², Gabriela Daszkiewicz², Maria Respondek-Liberska ${ }^{1}$

\author{
${ }^{1}$ Department for Foetal Malformation Diagnosis and Prevention, Medical University \\ of Lodz, Lodz, Poland \\ ${ }^{2}$ Student's Scientific Association "Prenatal Cardiology", Medical University of Lodz, \\ Lodz, Poland \\ ${ }^{3}$ Department of Paediatrics, Oncology, and Haematology, Medical University of Lodz, \\ Lodz, Poland
}

Submitted: 29 October 2020

Accepted: 25 January 2021

Arch Med Sci

DOI: https://doi.org/10.5114/aoms/132778

Copyright $\odot 2021$ Termedia \& Banach

\section{Abstract}

Introduction: The placental thickness (PTh) is an ultrasonographic measurement commonly used to assess the placenta. The study aimed to determine selected factors influencing PTh in 2D prenatal ultrasonographic examination. It might have a special value in difficult cases for interpretation when PTh is above or below the reference values.

Material and methods: In this retrospective study, we analysed the results of foetal ECHO examination of 2833 foetuses performed between June 2016 and December 2019 in our single unit. 596 healthy foetuses older than 12 weeks of gestation from singleton pregnancies were enrolled in the study. The following parameters were used in the further analysis: placental implantation site, gestational age according to the last menstrual period (LMP) and foetal biometry (FB); maternal weight, height, and body mass index (BMI) at the time of examination; and PTh.

Results: PTh was affected by its location: posterior $33 \mathrm{~mm}$ vs. anterior $30 \mathrm{~mm}$ $(p<0.001)$. Moreover, its thickness significantly correlated with gestational age according to FB $(r=0.386, p<0.001)$, LMP $(r=0.369, p<0.001)$, maternal weight $(r=0.192, p<0.001)$, height $(r=0.125, p=0.002)$, and BMI $(r=$ $0.147, p<0.001)$, but not with maternal age $(r=0.050, p=0.219)$. A linear regression model based on these data explained the $16.38 \%$ variability of the tested subjects $(p<0.001)$.

Conclusions: Our observations suggest that maternal weight correlated more strongly with PTh than maternal BMI. For PTh evaluation, it is important to pay attention to the placental implantation site - the posterior placenta was thicker than the anterior placenta. Moreover, PTh variability remains largely unknown; therefore, further research in this field is needed.

Key words: ultrasonography, gestational age, obstetrics, maternal BMI, placental thickness, maternal weight.

\section{Introduction}

Placental features describing its wellbeing are among the crucial factors influencing pregnancy outcomes. Placental morphology, implantation site, and thickness are a few such features that may be assessed during prenatal ultrasound examination [1, 2]. Placental thickness (PTh)

\author{
Corresponding author: \\ Filip F. Karuga \\ Student's Scientific \\ Association "Prenatal \\ Cardiology" \\ Medical University \\ of Lodz \\ Lodz, Poland \\ Phone: +48 422725445 \\ E-mail: filipfranciszek439@ \\ gmail.com
}


seems to be the easiest placental measurement, which can be taken during ultrasound examination; however, its interpretation is more complex [3]. It was previously proven that an abnormally thin or thick placenta leads to an increased prevalence of perinatal morbidity and mortality. In the year 1985, a placenta thicker than $4 \mathrm{~cm}$ was considered to be abnormal and was associated with worse pregnancy outcomes [4]. Various studies reported that factors like maternal body mass index (BMI) or placental implantation site significantly influence placental morphology and the cut-off value for PTh nomograms [3, 5, 6]. There are also several studies that associated PTh with neonatal outcome [1], neonatal birth weight [7], neonatal hypotrophy, risk of maternal preeclampsia, and other complications [8, 9] (Figure 1).

\section{Aim}

We aimed to determine the factors influencing PTh. Deeper insight into this topic may facilitate appropriate PTh interpretation.

\section{Material and methods}

We retrospectively analysed the results of the first foetal ECHO examinations of 2833 foetuses, performed between June 2016 and December 2019 at our unit. We enrolled 596 foetuses older than 12 weeks of gestation in the study. Only singleton healthy foetuses delivered at term (according to the last menstrual period (LMP)) in our hospital and discharged within 3-4 days were included in the study. Exclusion criteria were as follows: gestational diabetes, hydrops fetalis or any other foetal cardiac or extracardiac anomalies, difference between gestational age based on LMP and foetal biometry (FB) $>10$ days, amniotic fluid index $<8 \mathrm{~cm}$ or $>22 \mathrm{~cm}$, and premature delivery (<37 weeks of gestation).

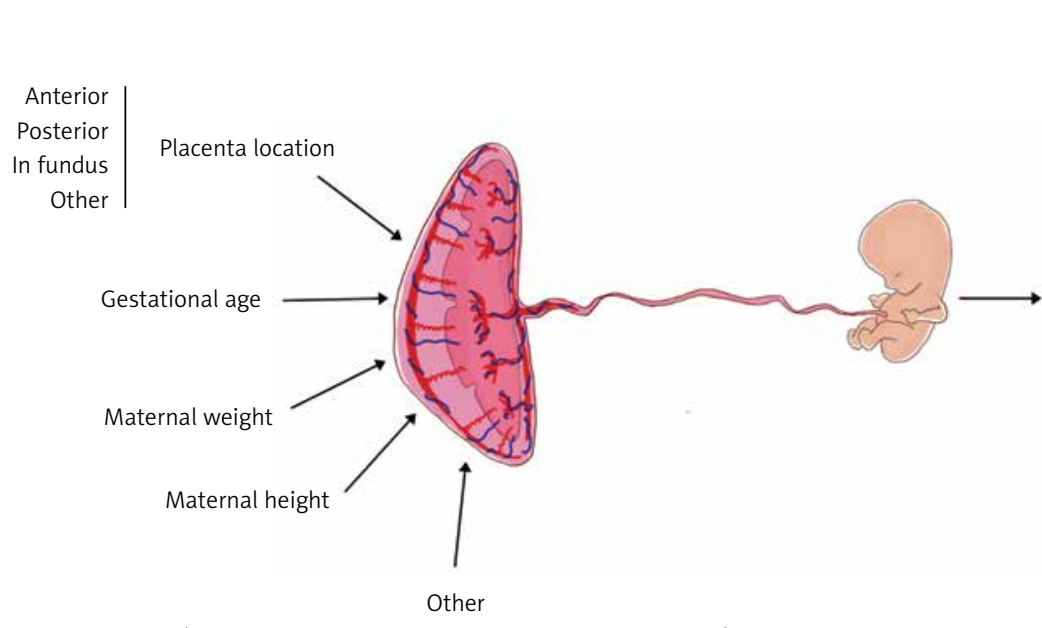

(smoking status, race, diabetes, hypertensive disease)

Figure 1. Schematic representation of risk factors for abnormal placental thickness and related complications
The following parameters were evaluated: placental implantation site, PTh, gestational age (GA) according to $L M P$ and $F B$, and maternal weight, height, and BMI at the time of examination. PTh was measured perpendicularly to the chorionic plate in its largest dimension (Figure 2). The measurements were taken by 4 professionals, experienced in prenatal diagnostics from the same single unit. All physicians in our team performed the ultrasound examinations in a unified manner, and of each exam was stored digitally [10].

\section{Statistical analysis}

All the results were validated and accepted by the head of our Department on a daily or weekly basis. None of the aforementioned parameters had a normal distribution; therefore, we used proper nonparametric tests available in Statistica 13.1 PL (StatSoft, Tulsa, USA) and presented the data as median with interquartile range (IQR). Written consent to use and process the medical data was obtained from all included patients.

\section{Results}

\section{Clinical group characterization}

The median maternal age was 31 years (IQR: 27-35 years). The patients visited our department at a median gestational age of 25 weeks (IQR: 21.3-28.4 weeks) according to LMP. Anterior placenta was found in 328 (55\%) pregnant women, whereas 250 (42\%) had posterior placenta. Nine $(1.5 \%)$ placentas were found in fundus of the uterus, and 9 (1.5\%) had another location. All placentas had appropriate vascularity according to the ultrasound examination: normal foetal biometry and normal Doppler blood flow in umbilical arteries and vein. The median PTh was $30 \mathrm{~mm}$ (IQR: 25-38 mm).

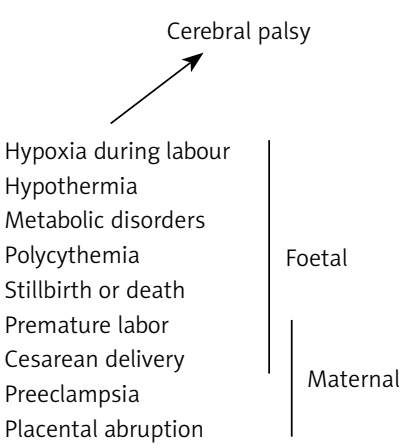

Placental abruption 



Figure 2. Placental thickness measurement technique during ultrasound examination. A - Placental thickness within normal limits, despite maternal body weight $111 \mathrm{~kg}$. B - Increased placental thickness and maternal body weight of $91.5 \mathrm{~kg}\left(\mathrm{BMI} 34 \mathrm{~kg} / \mathrm{m}^{2}\right)$. Difficult standardization of measurement due to significantly increased placental thickness

\section{Parameters affecting placental thickness}

PTh significantly correlated with gestational age according to both FB $(r=0.386, p<0.001$, Figure 3 B) and LMP $(r=0.369, p<0.001$, Figure 3 C); maternal weight $(r=0.192, p<0.001$, Figure $3 \mathrm{D})$, height $(r=0.125, p=0.002$, Figure $3 \mathrm{E})$, and BMI $(r=0.147, p<0.001$, Figure $3 \mathrm{~F})$, but not with maternal age $(r=0.050, p=0.219$, Figure $3 \mathrm{~A})$. Placental location significantly affected its thickness ( $p<0.001$, Kruskal-Wallis test) (Figure 4). The median PTh was $42 \mathrm{~mm}$ (IQR: 30$49 \mathrm{~mm}$ ) for placentas located in the fundus of the uterus, $33 \mathrm{~mm}$ (IQR: 26.8-40 $\mathrm{mm}$ ) for posterior placentas, $30 \mathrm{~mm}$ (IQR: 23.7-35 mm) for anterior placentas, and $27 \mathrm{~mm}$ (IQR: 25-30 mm) for placentas placed in other locations. Significant dependencies were observed between posterior and anterior placentas ( $p<0.001$, UMW) as well as between anterior placentas and those localized in the fundus $(p=0.034)$.

\section{Linear regression model}

A linear regression model (recurrent progressive steps) based on the data explains only $16.38 \%$ of the variability of the tested phenomena $(p<0.001)$ : FB $(p<0.001)$, placenta location (anterior vs. posterior, $p<0.001)$, maternal weight $(p=0.018)$, and height $(p=0.021)$.

\section{Discussion}

During routine ultrasound examination it is possible to assess various information about foetal and placental parameters. Obstetricians and sonographers are aware of the importance of analysing the placental location, especially its lower segment [11], and about the possibilities of placenta accreta spectrum - placenta creta, increta, and percreta [12]. As well as the placental location, PTh is another feature that can be assessed almost effortlessly during ultrasound examination. PTh measurement is a simple and widely used technique that may provide specialists with substantial knowledge. Despite the fact that placentas and PTh are being broadly investigated, specific maternal factors affecting this parameter have not yet been assessed in numerous groups of pregnant women [13-16].

In our study, we focused only on PTh because it is a very simple method and does not extend the time of examination. Moreover, PTh measurement did not require sophisticated software like, for instance, in the case of $3 \mathrm{D}$ reconstruction. We tried to analyse how particular parameters (FB, MA, LMP, mother's BMI, mother's body weight, and mother's height) affect the PTh value, in order to assess which factors have a significant influence on PTh and how they affect PTh interpretation. Due to the retrospective character of the study, not all the possible factors influencing foetal homeostasis, such as genetic factors, were taken into consideration $[1,7,17,18]$.

As early as in 1985 it was indicated that PTh increases with advancing GA [4]. Depending on the study, the correlation between PTh and GA is significantly different. The study of Azagidi et al. reported the most significant strong positive correlation in the $2^{\text {nd }}$ trimester $(r=0.794)$. Results suggest that PTh has a linear relationship with GA, sonographically estimated foetal weight, and foetal growth parameters. Therefore, PTh can be used along with other foetal growth parameters to predict GA more accurately, which may be especially useful when a pregnant woman is not sure or does not know her LMP [19] or there was no 1st trimester scan. Agwuna et al. reported almost the same correlation value for the $2^{\text {nd }}$ trimester; $r=0.791$, $p=0.01$ [20]. In a cross-sectional prospective study performed on a group of 211 pregnant women by Karthikeyan et al. similar findings where determined. The statistical analysis revealed $r=0.609$, $r=0.812$, and $r=0.814$ for the $1^{\text {st }}, 2^{\text {nd }}$, and the $3^{\text {rd }}$ trimesters, respectively, and the $p$-value was $<0.001$ [21]. In the paper published by Keshavarz et al., 
A

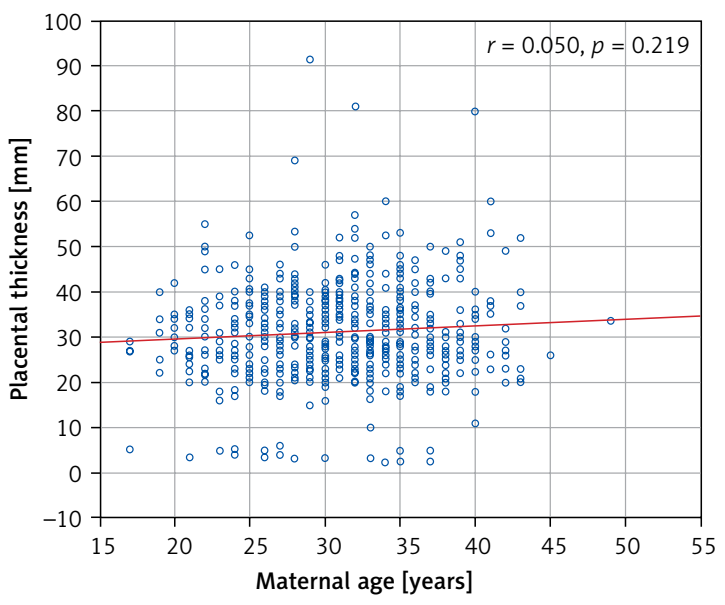

C

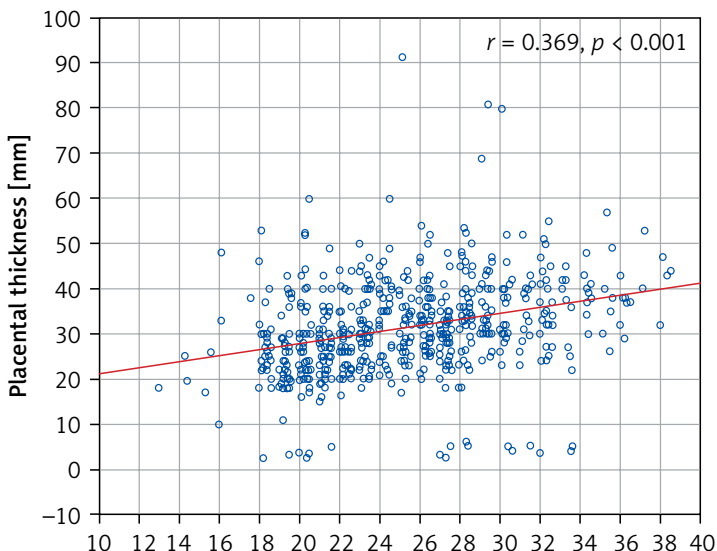

Gestational age according to the last menstrual period [weeks]

E

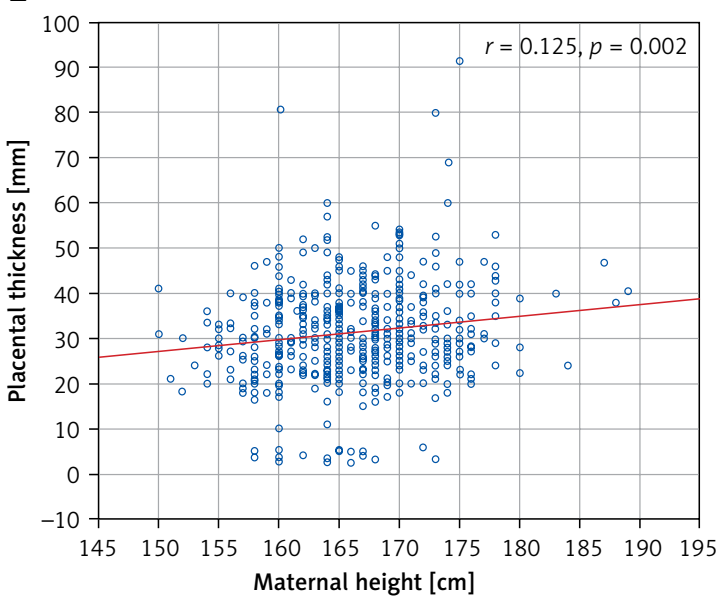

B

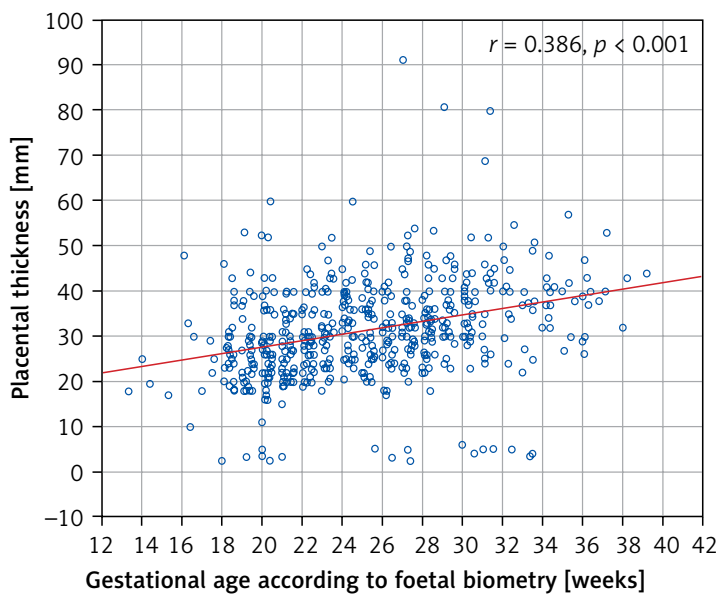

D

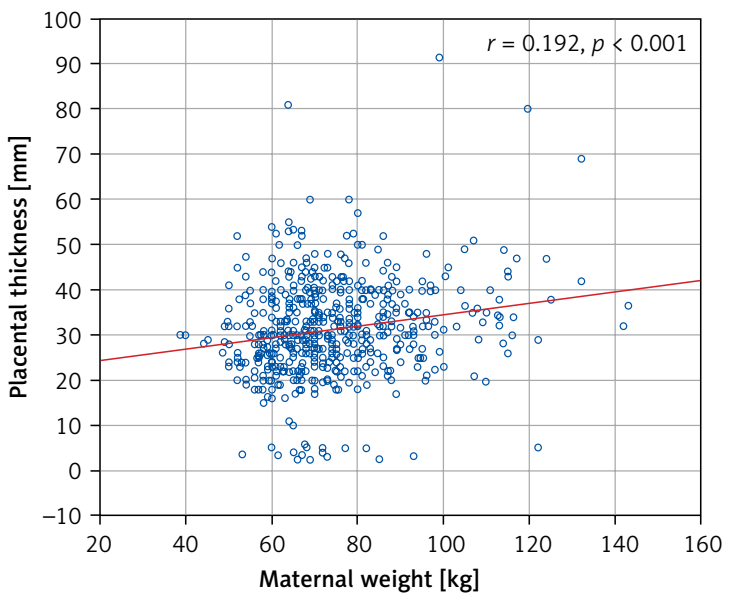

$\mathrm{F}$

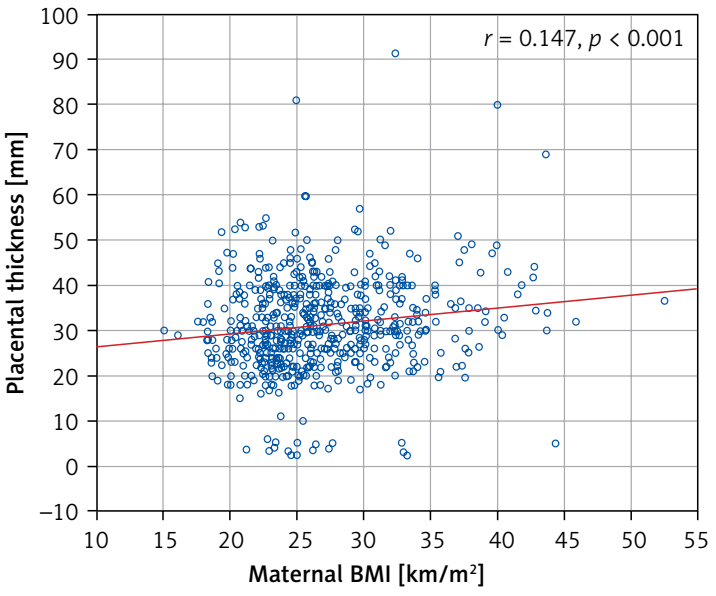

Figure 3. Correlation between placental thickness and tested continuous variables: maternal age (A), foetal biometry $(B)$, gestational age according to LMP $(\mathbf{C})$, maternal weight $(D)$, maternal height $(E)$, and maternal BMI $(F)$

in which placental thickness was measured among 400 healthy pregnant women, the authors found even stronger positive linear correlation between GA and PTh ( $p<0.001 ; r=0.93$ ) [22]. Our study confirmed the presence of positive correlation between PTh and GA, however, the described correlation was substantially weaker according to both FB and LMP ( $r=0.386$ and $r=0.369$, respectively). There are several reports that also indicate that the described correlation is not that strong. In the study performed on 3183 pregnant women between the $16^{\text {th }}$ and $40^{\text {th }}$ week of gestation the slope of the curve was smaller than in the studies described before and more similar to the results 
from our study [9]. Similar curves were found on the figures presenting the data obtained from 114 prenatal ultrasounds from the study of Lee et al. [3]. In the study of Verma et al. the correlation between PTh and GA in the $33^{\text {rd }}$ to $40^{\text {th }}$ week was established at the level of $r=0.386$ $(p<0.001)$; however, between the $19^{\text {th }}$ and $32^{\text {nd }}$ week of gestation this figure was $r=0.792$, and the observed correlation was similar to those reported by Azagidi et al. and Agwuna et al. [23].

There are parameters that indicate a much clearer correlation between themselves and GA comparing with PTh - for example: abdominal circumference, transversal diameter of the foetal heart, or thymus circumference [24-26]. Papers regarding the correlation between PTh and GA in the ultrasound examination present varied data, but they all agree that there is a positive correlation. This knowledge can be used to predict GA and progress in foetal growth.

The relation between increased mother's $\mathrm{BMI}$ and the foetal overgrowth is described in various papers. In the meta-analysis by Gaudet et al. maternal obesity played a significant role in the development of macrosomic newborns $(\mathrm{OR}=2.17,95 \% \mathrm{Cl}: 1.92-2.45)$ [27]. The correlation between BMI and PTh has not been so broadly investigated. Baptiste-Roberts et al. found that with increased pre-pregnancy BMI and pregnancy weight gain, the likelihood of placental hypertrophy increases [28]. Kiliopa et al. described the positive correlation between maternal BMI and PTh $(r=0.42 ; p<0.001)$ [5]. In our research the correlation between $\mathrm{BMI}$ and PTh was more than 2 times lower $r=0.147, p<0.001$. Additionally, we found that maternal weight correlates better $(r=$ $0.192, p<0.001$ ) with the PTh than maternal BMI.

In our study we have also analysed how placental implantation site affects PTh value. It turned out, as other studies also confirm, that PTh varies depending on the placental location [3, 29]. We have demonstrated that the thickest placentas are usually found in the fundus of the uterus (median PTh was $42 \mathrm{~mm}$ ). Placentas with posterior location were thinner by $9 \mathrm{~mm}$ than the placentas located in the fundus. Placentas with anterior location presented the lowest PTh values $(30 \mathrm{~mm})$ and were thinner by $3 \mathrm{~mm}$ compared with placentas located at the posterior wall of the uterus. In the study of Lee et al. the anteriorly located placentas were also thinner than the ones implanted to the posterior wall or fundus; however, the difference was $7 \mathrm{~mm}$ [3]. Our findings also agree with the study by Durnwald et al., in which placentas located in the fundus were the thickest and those located at the anterior wall were the thinnest [29]. In the study of Menon et al. the association between placental location and PTh was found to be not significant [30].

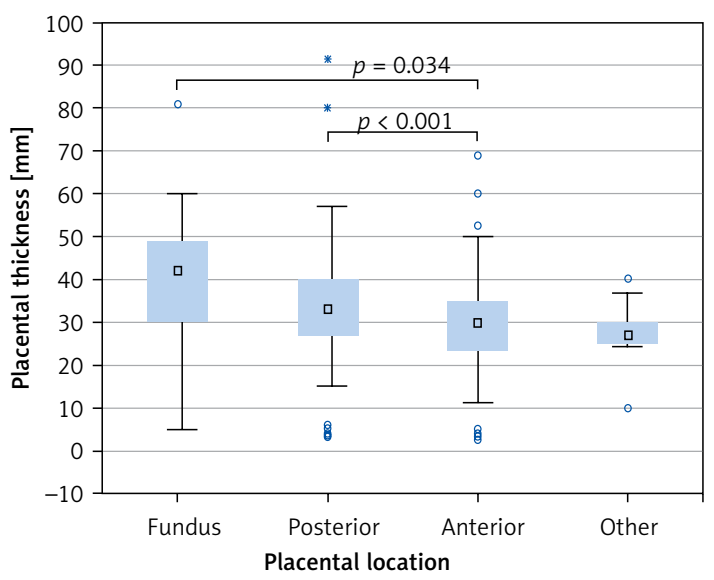

Figure 4. Placental location affecting its thickness ( $p<0.001$, Kruskal-Wallis test)

Although many authors underlined the benefits which may result from the measurement of the PTh, there are no recommendations in ISUOG guidelines to report this parameter during standard prenatal ultrasound examination [31]. We anticipate that PTh measuring should be routinely included in every prenatal ultrasound examination because it is easy to perform and may help to confirm foetal wellbeing. Our research could be of special value in difficult cases for interpretation, for example, when the PTh is above or below the reference value or in the case of underweight or overweight pregnant women.

\section{Strengh and limitation}

The strengths of this study are as follows:

- validation of one of the most simple approaches to assess the placental size,

- sufficient study group size $(n=596)$,

- small number of publications regarding the factors that influence PTh.

The limitations of this study are as follows:

- subjective character of ultrasound PTh examination,

- retrospective character of the study,

- other established parameters affecting placenta size, e.g. gravidity, parity, genetic factors, maternal health status, and habits, were not taken into consideration in this study.

\section{Conclusions}

Our observations suggest that maternal weight correlates more strongly with PTh than maternal BMI. In PTh evaluation it is important to pay attention to the placental implantation site - posterior placenta was thicker than the anterior placenta. Moreover, PTh variability remains largely unknown; therefore, further research in this field is needed. 


\section{Acknowledgments}

We would like to thank all the patients of the Department of Prenatal Cardiology, Polish Mother's Memorial Hospital (PMMH), Lodz, Poland, for their participation in our study. We would also like to thank the staff at the Department of Prenatal Cardiology $\mathrm{PMMH}$ for their input into this research, especially to Prof. M. Słodki MD, PhD, J. Murlewska MD, PhD, A. Żalińska MD, L. Sokołowski MD, and I. Pietrzyk, BS.

\section{Conflict of interest}

The authors declare no conflict of interest.

\section{References}

1. Nagpal K, Mittal P, Grover SB. Role of ultrasonographic placental thickness in prediction of fetal outcome: a prospective indian study. J Obstet Gynecol India 2018; 68: 349-54.

2. Fadl S, Moshiri M, Fligner CL, Katz DS, Dighe M. Placental imaging: normal appearance with review of pathologic findings. Radiographics 2017; 37: 979-98.

3. Lee AJ, Bethune M, Hiscock RJ. Placental thickness in the second trimester. J Ultrasound Med 2012; 31: 213-8.

4. Hoddick WK, Mahony BS, Callen PW, Filly RA. Placental thickness. J Ultrasound Med 1985; 4: 479-82.

5. Kiliopa EO, Gesase PA, Lilungulu A. The effects of maternal body mass index on placental morphology and foetal birth weight: a study from Dodoma Central Region, Tanzania. South Sudan Med J 2019; 12.

6. Karunakaran I, Nalinakumari SD, Ponniraivan K. Effect of increased prepregnancy body mass index on placental morphologic features in gestational diabetes. J Clin Diagnostic Res 2018; 12: AC13-7.

7. Ahn KH, Lee JH, Cho GJ, Hong SC, Oh MJ, Kim HJ. Placental thickness-to-estimated foetal weight ratios and small-for-gestational-age infants at delivery. J Obstet Gynaecol 2017; 37: 883-7.

8. Vachon-Marceau C, Demers S, Markey S, et al. Bujold first-trimester placental thickness and the risk of preeclampsia or SGA. Placenta 2017; 57: 123-8.

9. Miwa I, Sase M, Torii M, Sanai H, Nakamura Y, Ueda K. A thick placenta: a predictor of adverse pregnancy outcomes. Springerplus 2014; 3: 353.

10. Karuga FF, Szmyd B, Respondek-Liberska M. Fetal congenital heart disease and fetal position - are they related? Prenat Cardiol 2019; 9: 33-6.

11. Bhide A, Laoreti A, Kaelin Agten A, et al. Lower uterine segment placental thickness in women with abnormally invasive placenta. Acta Obstet Gynecol Scand 2019; 98 : 95-100.

12. Jauniaux E, Collins S, Burton GJ. Placenta accreta spectrum: pathophysiology and evidence-based anatomy for prenatal ultrasound imaging. Am J Obstet Gynecol 2018; 218: 75-87.

13. Słabuszewska-Jóźwiak A, Włodarczyk M, Ciebiera M, et al. Placental DNA methylation in caesarean sections - a pilot study. Arch Med Sci DOI: https://doi. org/10.5114/aoms.2020.95422.

14. Xia F, Meng Q, Zhang F, et al. IncRNA SNHG6 improves placental villous cell function in an in vitro model of gestational diabetes mellitus. Arch Med Sci DOI: https:// doi.org/10.5114/aoms.2020.100643.
15. Kornacki J, Wender-Ożegowska E. Utility of biochemical tests in prediction, diagnostics and clinical management of preeclampsia: a review. Arch Med Sci 2020; 16: 1370-5.

16. Wang L, Li Y. MiR-29b-3p affects growth and biological functions of human extravillous trophoblast cells by regulating bradykinin B2 receptor. Arch Med Sci DOI: https://doi.org/10.5114/aoms.2019.91512.

17. Szmyd B, Karuga F, Gach A, Moszura T, Kopala M, Respondek-Liberska M. Complex cardiovascular defects in a male infant with Williams syndrome juxtaposed with the results of a preliminary survey illustrating other patients' outcomes. Kardiol Pol 2021. DOI: 10.33963/ KP.15740.

18. Gheorghe CP, Goyal R, Mittal A, Longo LD. Gene expression in the placenta: maternal stress and epigenetic responses. Int J Dev Biol 2010; 54: 507-23.

19. Azagidi A, Ibitoye B, Makinde O, Idowu B, Aderibigbe A. Fetal gestational age determination using ultrasound placental thickness. J Med Ultrasound 2020; 28: 17-23.

20. Agwuna K, Eze C, Ukoha P, Umeh U. Relationship between sonographic placental thickness and gestational age in normal singleton fetuses in Enugu, Southeast Nigeria. Ann Med Health Sci Res 2016; 6: 335-40.

21. Karthikeyan T, Subramaniam RK, Johnson WMS, Prabhu K. Placental thickness and its correlation to gestational age and foetal growth parameters - a cross sectional ultrasonographic study. J Clin Diagnostic Res 2012; 6: 1732-5.

22. Keshavarz E, Motevasselian M, Amirnazeri B, et al. Gestational age-specific reference values of placental thickness in normal pregnant women. Women Heal 2019; 59: 718-29.

23. Verma P, Gupta M, Verma S. Correlation of placental thickness with gestational age in antenatal women. Int J Reprod Contracept Obs Gynecol 2019; 8: 3061-6.

24. Adeyekun AA, Orji MO. Predictive accuracy of transcerebellar diameter in comparison with other foetal biometric parameters for gestational age estimation among pregnant Nigerian women. East Afr Med J 2014; 91: 138-44.

25. Sylwestrzak O, Respondek-Liberska M. Echocardiographic metods of fetal heart size assessment-heart to chest area ratio and transversal heart diameter. Prenat Cardiol 2018; 8: 20-3.

26. Zych-Krekora K, Krekora M, Słodki M, et al. Nomograms of the fetal thymus for clinical practice. Arch Med Sci DOI: https://doi.org/10.5114/aoms.2019.86189.

27. Gaudet L, Ferraro ZM, Wen SW, Walker M. Maternal obesity and occurrence of fetal macrosomia: a systematic review and meta-analysis. Biomed Res Int 2014; 2014: 640291.

28. Baptiste-Roberts K, Salafia CM, Nicholson WK, Duggan A, Wang NY, Brancati FL. Maternal risk factors for abnormal placental growth: The national collaborative perinatal project. BMC Pregnancy Childbirth 2008; 8: 44.

29. Durnwald C, Mercer B. Ultrasonographic estimation of placental thickness with advancing gestational age. Am J Obstet Gynecol 2004; 191: 178.

30. Menon M. Gestational age determination by ultrasonic placental thickness measurement. Orig Res Artic Indian J Obstet Gynecol Res 2016; 3: 279-82.

31. Salomon LJ, Alfirevic Z, Da Silva Costa F, et al. ISUOG Practice Guidelines: ultrasound assessment of fetal biometry and growth. Ultrasound Obstet Gynecol 2019; 53: 715-23. 